Document downloaded from:

http://hdl.handle.net/10251/103263

This paper must be cited as:

Alfaro Saiz, JJ.; Verdecho Sáez, MJ.; Rodríguez Rodríguez, R. (2013). How to achieve dynamic and flexible performance management systems for collaborative processes. IFIP Advances in Information and Communication Technology. 408:639-647. doi:10.1007/978-3642-40543-3_67

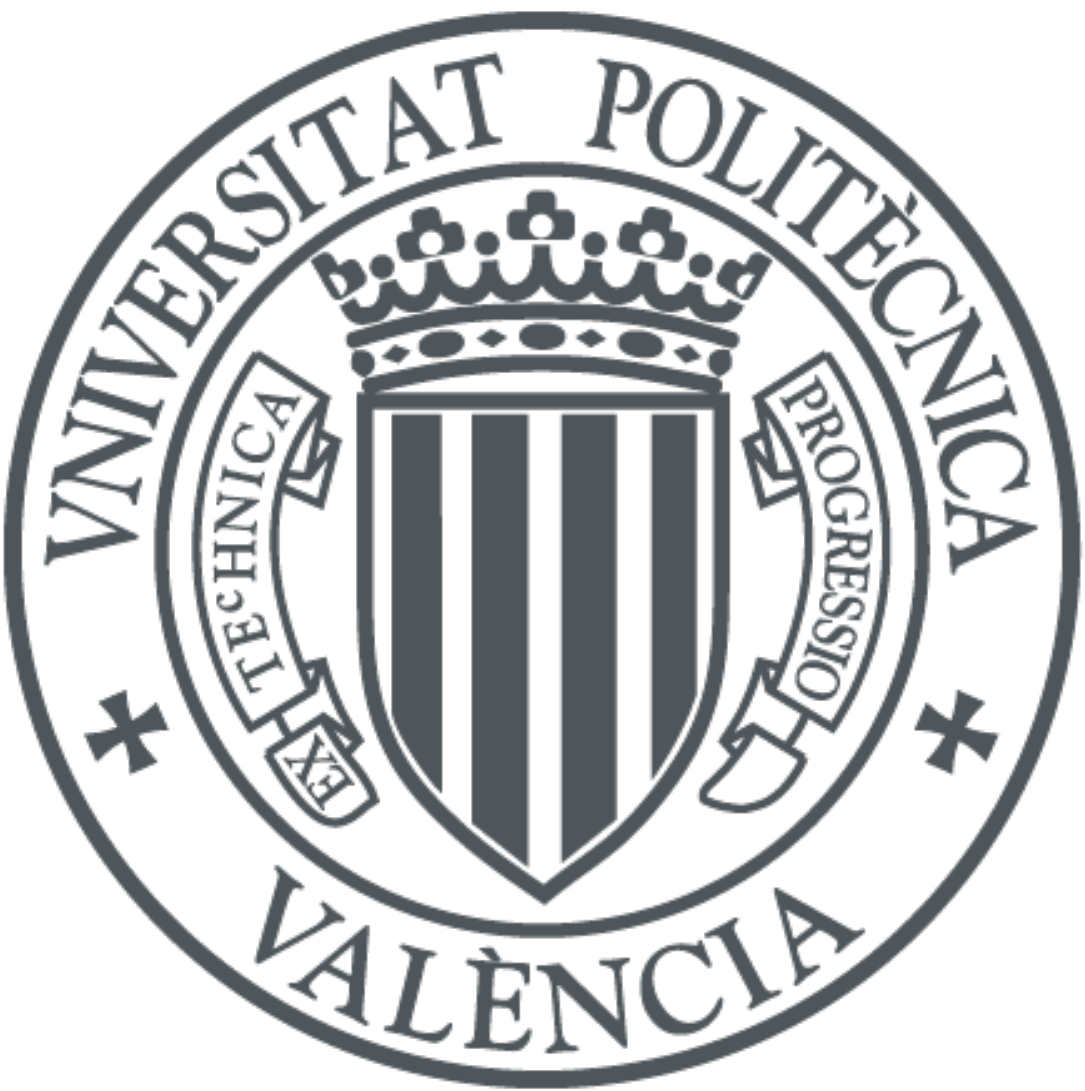

The final publication is available at

https://doi.org/10.1007/978-3-642-40543-3_67

Copyright Springer

Additional Information 


\title{
How to achieve dynamic and flexible Performance Management Systems for collaborative processes
}

\author{
Juan-Jose Alfaro-Saiz', , María-José Verdecho-Saez ', Raúl Rodríguez-Rodríguez ', \\ ${ }^{1}$ Centro de Investigación, Gestión e Ingeniería de Producción, Universidad Politécnica de Valencia, Edificio 7D, Camino de \\ Vera, s/n, Valencia 46022, España, jalfaro@omp.upv.es
}

\begin{abstract}
One of the main characteristics of successful collaborative networks is their ability to continuously reformulating their processes. The, they can quickly get adapted to environment needs and therefore define more competitive processes. One of biggest weaknesses of Performance Management Systems (PMS) when dealing with collaborative processes associated to collaborative networks, is their low degree of both dynamicity and flexibility to get adapted to the changes that such collaborative processes experiment. The main changes that can take place are, among others, small or large modifications of the own processes, entrance and/or exit of new participants in the process, incorporation and/or elimination of processes, changes in objectives and/or strategies of processes of some participant, or even of the whole collaborative network, etc. All this brings continuous modifications over the components that conform the PMS. Then, it is necessary to structure certain mechanisms that will provide with both dynamicity and flexibility to the PMS. Otherwise, these PMS will become obsolete in the short-time and will be not useful anymore. Further, these PMS will not measure properly performance and then they will become ineffective, becoming the source of troubles. Scientific literature shows that most of the works that deal with PMS dynamicity and flexibility are focused on intra-organizational contexts, leaving almost apart the collaborative networks ambit. This work analyses those aspects that prevent to the PMS to be dynamic and flexible when applied to manage the performance of collaborative networks. In addition, we discuss and analyse several mechanisms that should be incorporated into the PMS, making them more dynamic and flexible.
\end{abstract}

Keywords: Collaborative process, Dynamic and flexible, Performance management system

\section{Introduction}

Inter-organisational co-operation has been one of the most used organisational strategies to compete and become adapted to the exigencies of the global market. Thus, collaboration is becoming more a necessity than an option [1]. In this sense, collaborative business processes are the key components to be developed so that collaboration between companies is useful. For that reason, companies look for both organisational models and tools able to manage these processes at both inter and intra-organisational levels. In this context, it is necessary to measure the performance of these collaborative business processes in a twofold manner, from a global perspective (inter-enterprise) and from an individual or partial perspective (intra-enterprise). The collaborative business processes acquire an extended nature, in which two or more enterprises or organisations participate. Besides, "collaboration" is an amorphous meta-concept that has been interpreted in many different ways by both organisations and individuals, and when it is put in the context of the supply chain it needs yet further clarification [2]. So, when we talk about collaborative business processes, these are understood as "a process where two or more enterprises participate, independently of the degree of cooperation/collaboration existing between them". Figures 1 and 2 show a representation of this concept. Figure 1 shows four extended business processes belonging to three enterprises that are operating at the intra-organisational (white area) and inter-organisational (represented by a grid). 


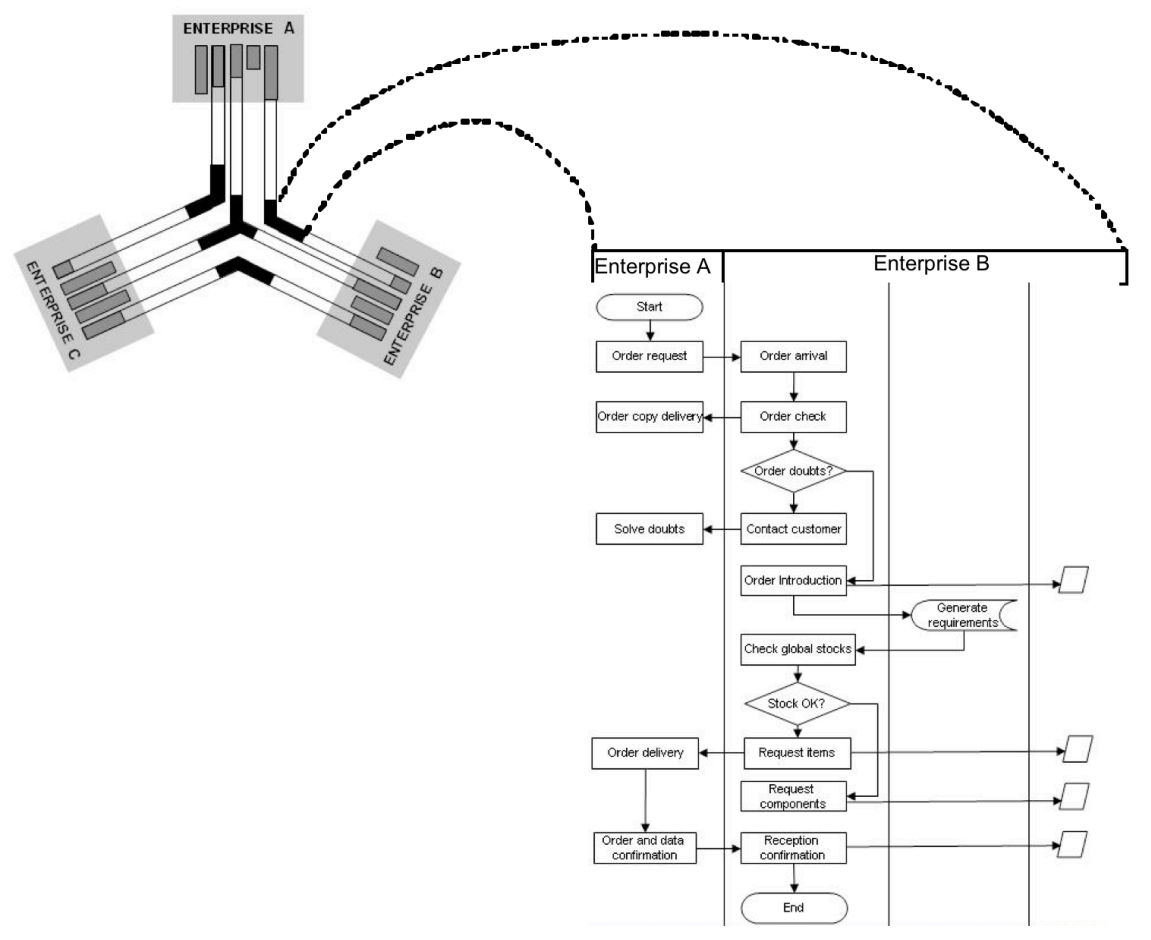

Fig. 1. Concept of collaborative business process

[3]

Figure 2 shows the relationship between the internal and the collaborative processes, where they may eventually need to exchange all kinds of resources, being usually necessary to share applications and data for their successful implementation.

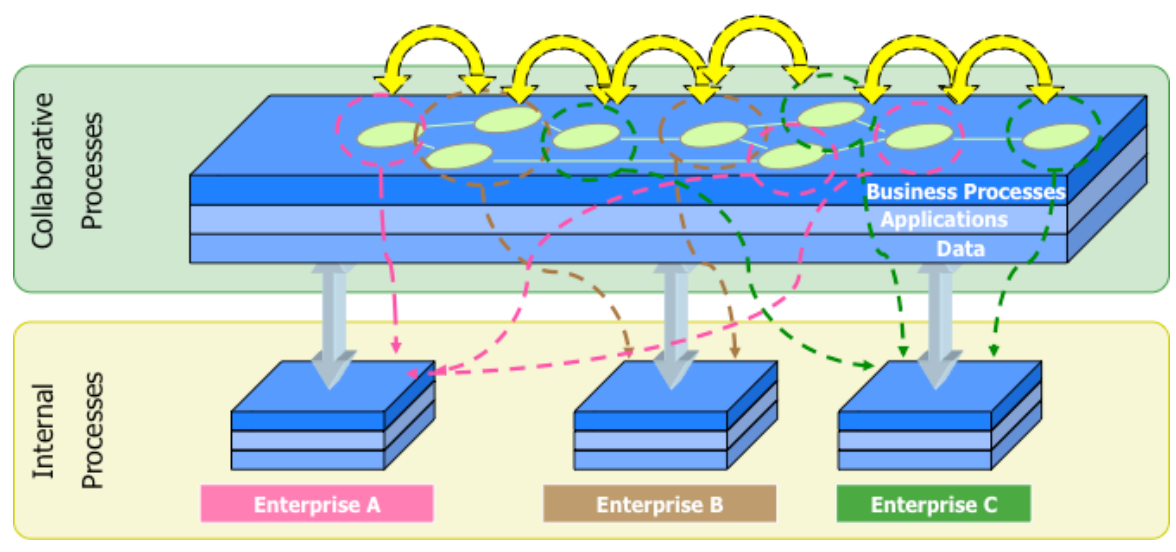

Fig. 2: Concept of collaborative business processes [4]

In the literature, most of the existing performance measurement systems (PMS) incorporate methodologies that are strictly rigid and that, therefore, do not favour an adequate adaptation to the changes that take place within collaborative organisations, which design together collaborative processes and where several organisations participate.

Then, it is necessary to structure certain mechanisms that will provide with dynamicity and flexibility to the PMS. Otherwise, these PMS will become obsolete in the short-time and will be not be useful anymore. Further, these PMS will not measure properly performance and then they will become ineffective, being the source of troubles.

In the next section of this paper we review the literature to analyze the works that address the dynamic issue within PMS, then it will describe the aspects that prevent a PMS to be dynamic and flexible when dealing with collaborative processes; later it will address the mechanisms to provide flexibility and dynamism to the PMS operating in that context, and finally, it highlights the main conclusions. 


\section{Literature review}

Although many authors have suggested the importance of designing dynamic PMS, few have deepened on how to define mechanisms to provide them with that quality. Furthermore, almost all of the works have focused on the intraorganizational context. This section (see Table I) presents a brief summary of the most interesting contributions found in the literature, which either directly or indirectly serves to address the dynamism and flexibility concepts within a PMS that deals with business processes of a $\mathrm{CN}$.

\begin{tabular}{|c|c|c|}
\hline Year & Author & Main contribution \\
\hline 1997 & $\begin{array}{l}\text { Ghalayini et al. } \\
\text { [5] }\end{array}$ & $\begin{array}{l}\text { Developed the Integrated dynamic performance measurement system } \\
\text { (IDPMS). This framework integrates three main areas of the company: } \\
\text { management, process improvement team, and factory shop floor. The IDPMS } \\
\text { synthesizes three existing tools (PMQ, half-life concept, VFCT diagrams). } \\
\text { The IDPMS provides an integrated approach that supports performance } \\
\text { measurement system alignment across managerial and operational levels. }\end{array}$ \\
\hline 1999 & $\begin{array}{l}\text { Waggoner et al. } \\
{[6]}\end{array}$ & $\begin{array}{l}\text { Created a framework about the forces impacting performance measurement } \\
\text { system evolution and change. Established four groups: internal influences, } \\
\text { external influences, process issues and transformational issues. }\end{array}$ \\
\hline 2000 & Bititci et al. [7] & $\begin{array}{l}\text { Created a model (the integrated model) in which for different levels (business, } \\
\text { business units and business processes) it applies a structure based in an } \\
\text { external and internal monitoring system that helps to review the objectives } \\
\text { and KPIs for deployment and alignment. } \\
\text { Focusing on exploring the use of IT based management tools. This author } \\
\text { affirms that the requirements from a dynamic PMS are divided into two } \\
\text { categories: } \\
\text { A) requirements of the own framework. } \\
\text { B) The requirements for an IT platform. }\end{array}$ \\
\hline 2003 & $\begin{array}{l}\text { Kennerley et al. } \\
{[8]}\end{array}$ & $\begin{array}{l}\text { Defined a list of enablers and barriers to the evolution of PMS, establishing } \\
\text { also the principal internal and external triggers that might affect to the PMS. } \\
\text { Besides, it groups together to the enablers and barriers under four critical } \\
\text { factors: culture, process, people and systems. }\end{array}$ \\
\hline 2003 & $\begin{array}{l}\text { Kennerley \& } \\
\text { Neely [9] }\end{array}$ & $\begin{array}{l}\text { Derived from the previous work, they developed a Framework of factors } \\
\text { affecting the evolution of PMS. This framework is based on the fact that the } \\
\text { evolution of a system is possible through execution of four phases, namely use } \\
\text { (of the PMS), reflection (to identify where it is no longer appropriate and } \\
\text { where enhancements need to be made), modification (to ensure alignment to } \\
\text { the organisation's new circumstances. and deployment (of the modified PMS). }\end{array}$ \\
\hline 2005 & Najmi et al. [10] & $\begin{array}{l}\text { Designed a Performance Measurement System Review Framework, on the } \\
\text { one hand reviews the business performance at three levels (on-going review, } \\
\text { periodic review and overall review) and on the other, the PMS performance } \\
\text { review (focuses on the actions taken by the PMS, being of special relevance } \\
\text { the PMS design impact analysis y el PMS implementation impact). }\end{array}$ \\
\hline 2011 & $\begin{array}{l}\text { Salloum M. } \\
{[11]}\end{array}$ & $\begin{array}{l}\text { Developed a framework for dynamic PMS (See Figure } 3 \text { ). The framework } \\
\text { incorporates the factors that constitute dynamics in a PMS, their systemisation } \\
\text { and what to consider when realizing them in practice. It uses } 19 \text { factors that } \\
\text { are systemized into five sub-headings (review process, IT-Systems, } \\
\text { management, employees and culture). PMS. Additionally, in order to realise } \\
\text { dynamic PMS in practice, two broad factors need to be considered: A) the } \\
\text { PMS design: the characteristics of the current PMS and the systems and } \\
\text { processes integrated with it; } \\
\text { B) the PMS context: the current IT-system capabilities and the level of } \\
\text { maturity of management, employees and culture in the organisation. }\end{array}$ \\
\hline
\end{tabular}

Table I 


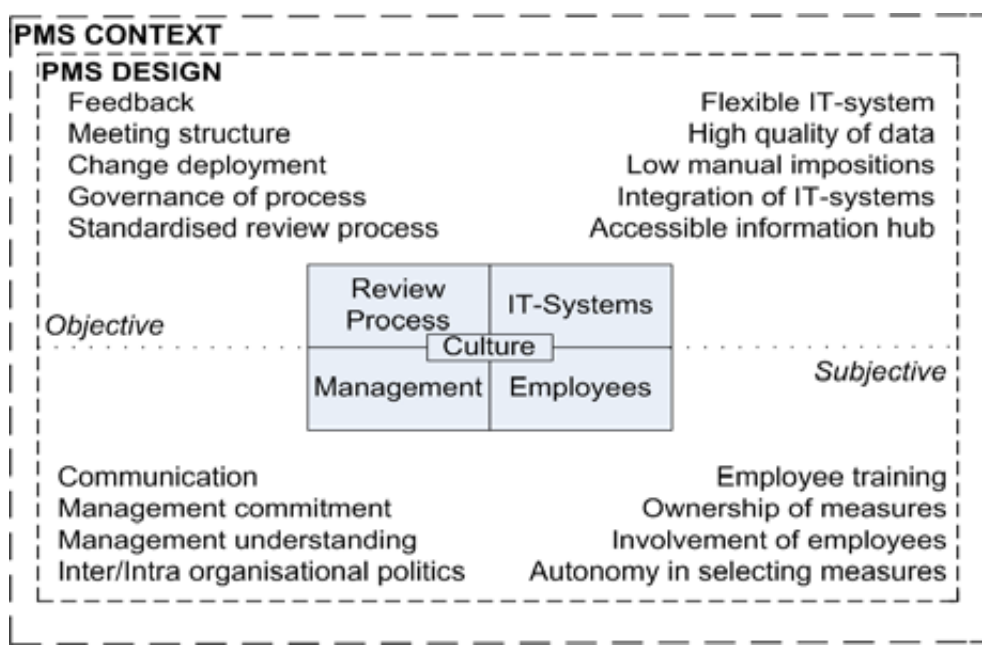

Figure 3: Framework for dynamic PMS [11]

\section{Aspects that prevent PMS of being dynamic and flexible in the context of Collaborative Processes}

Currently, collaborative business processes have a set of characteristics that prevent them from being properly managed. Thus, PMS found difficult to get adapted to such characteristics and therefore do not carry out a proper management of the processes. Those features are described next:

A) Implication of various actors. In a collaborative business processes context, there are at least two companies involved, but it is not common to find collaborative processes crossing four, five or more companies. When designed, implemented and put into operation, a collaborative process must be aware of all the components that influence it: operations, resources, applications, information systems, etc. Any change in one of these components may affect the collaborative process performance and this fact should be registered in the PMS, keeping it in mind when referring to the collaborative process performance. Further, it may objectively assess whether the results are correct or they have been affected by this circumstance. Today, it is increasingly common that any of these components change quite often, because the participation in the collaborative process by multiple companies increases this possibility.

B) Lifecycle collaborative processes are becoming shorter. This feature is very important. Our current consumer society demands products and services that must be constantly renewed, in a rapid way. This circumstance forces companies to more quickly develop such products and services. The times spent on both the process engineering phase (definition, representation, design and construction) and on the operation phase (implementation and analysis) are becoming shorter. This fact, together with the number of actors involved in the collaborative process requires the used PMS to be very versatile, able to collect the singularities of both phases in the minimum time. With such short lifecycles, it is essential a perfect cooperation of all the collaborative network members.

C) Equity of resources risks and benefits. When several companies collaborate in a collaborative process, they must provide various types of resources (financial, material, human, etc.); so, it is necessary that the expected benefit they want to get is equitable to the investment risk, but especially equitable compared to other participants in the collaborative process. The applied PMS should be sensitive to this particularity, especially in very dynamic environments in which the creation or modification of processes is constant. Thus, transmitting a feeling of equity between partners is vital to the proper functioning of the collaborative process and, extensively, to maintain a good working environment within the $\mathrm{CN}$.

D) Information sharing. To properly work, collaborative processes need to share information, and this information has to be useful, meeting three key requirements: accuracy, timely and relevant. The role of IS/ICT is central to assure that the information exchange process is agile, effective and efficient. Moreover, when two or more companies share information, trust between them is essential for the collaborative process to be fully effective. 
E) Joint decision-making. One problem in collaborative processes contexts is the establishment of a consensus decision-making process, where the assignment of responsibilities is often a contentious element on many CN. Therefore, we must clarify the decision-making process, establishing the scope, level, participants, responsible and procedure. The decision process should be agreed by all the partners, and have to put the necessary resources to make it as dynamic as possible.

Once the main particularities of the collaborative processes have been described, the two main aspects that prevent PMS of being flexible and dynamic in this context are next analysed:

- Lack of a structured framework. Most of the PMS have a structure based on a methodology with several sequential steps, which describe how they will define a set of performance elements (objectives, KPIs, etc.) according to some criteria and hierarchies. The structure of these PMS is designed for relatively stable environments, where the modification of any of the performance elements involves a great effort and, in many cases, a rethinking of other involved downstream elements. Moreover, the methodologies are often tedious, with many phases and where the working groups are relatively homogeneous and therefore have not developed practical deployment scenarios for those working groups. Few PMS count with review mechanisms, and those that have them are thought to employ such mechanisms one or two times per year (totally inadequate regarding today's CN environments). In this sense, In this sense, when we apply the review mechanisms, in most cases, we are obliged to develop virtually all PMS methodology, which takes a lot of effort and time. The lack of traceability between performance elements, levels and participants within the collaborative process of the $\mathrm{CN}$ is a critical element that drives the PMS to be inefficient in these environments.

- Lack of integration between the PMS and intra/inter-organisational information systems. The performance management of any type of process requires a lot of information, but when we deal with collaborative processes the problem is not only the generation and processing of information itself, but also the different sources and actors involved in the generation of this information. Surely, if the PMS is not integrated into the information systems of the partners or at least they do not have the tools to connect to the PMS, the effectiveness and efficiency of the PMS will be lowered in a short time. Today, collaborative processes change so fast that necessitate the use of fully integrated PMS within the IS, at both the intra and inter-organisational context. The connection of all the performance elements of the PMS through the IS is a vital task in order to provide the PMS with both dynamicity and flexibility, which would enable that all the possible variations, modifications and adjustments to any of these elements have a rapid deployment by all partners. Currently, the available PMS for collaborative processes do not possess informational architectures to provide a proper and effective integration with ERP systems.

\section{Mechanisms to be incorporated into the PMS to be more dynamic and flexible}

This section describes the mechanisms and actions that should be incorporated into the PMS to provide them with both dynamism and flexibility. It is not intended to make a list of all of such mechanisms but rather to describe those which may have a greater role:

- To introduce mechanisms that trigger automatic PMS reviewing processes. Since changes in any of the PMS elements could not only be due to alterations of exogenous or endogenous events to the $\mathrm{CN}$, the reviews should be remembered automatically following a programmed pattern.

- To incorporate tools to analyse both the consistency and coherence between the performance elements. When one or more PMS elements are modified as a result of changes in the collaborative processes, the PMS tends to lose consistency and therefore its ability to manage the performance of such collaborative processes decreases. These tools can be based on consistency diagrams, cause-effect diagrams, some statistical techniques, etc.

- Simplify PMS methodologies. Sometimes PMS methodologies can be quite complex, which implies to be very laborious and then when any change occurs, the effect is that the PMS becomes a rigid tool, difficult to get adapted to the demands of collaborative processes.

- To define specialized working groups for reviewing and implementation (training). The implementation and maintenance of the PMS in collaborative environments requires the use of expert working groups of all partners involved. Thus, the higher the formation in this type of tools the greater the rapidity degree in applying the changes.

- Design and include within the PMS informational architectures, easy to integrate with the IS, both at the intra and inter-organizational context. Although many PMS are not associated (because are not developed yet) to any informational architecture that supports all of the necessary information for assuring a correct operation, within collaborative environments this is an essential factor. The use of different informational systems at different levels 
(intra and inter) requires from PMS to have an adequate information architecture that facilitates the integration of the system, establishing the mechanisms of standardization and compatibility and facilitating the collection and processing of information.

- Add KPIs monitoring systems. Monitoring systems are also very important. Here, it is necessary to improve the understanding of KPIs changes, and extensively how these affect to the collaborative processes performance. Different levels can be established depending on the type of information needed and the degree of information aggregation that different users need; obviously, the level of monitoring shall include the information required by the type of decision to be taken.

\section{Conclusions}

Collaborative business processes are the key components to be developed in order to achieve a useful and successful collaboration between companies. For that reason, it is necessary to measure the performance of these collaborative business processes in a twofold manner. Many authors have suggested the importance of designing dynamic PMS, but few have deepened on how to define mechanisms to provide them with that quality. This work has reviewed the aspects that prevent PMS of being both dynamic and flexible in the context of Collaborative Processes (implication of various actors, equity of resources, risks and benefits, shared information, collaborative processes lifecycle becoming increasingly shorter and joint decision-making). Thus, the two most important aspects preventing PMS of being dynamic and flexible in a collaborative context are: lack of a structured framework and lack of integration between the PMS and the intra/inter-organisational information systems. Finally, this work has commented the mechanisms that should be incorporated into the PMS to provide them with dynamism and flexibility. It is necessary to further research about these mechanisms to improve the PMS at the collaborative processes and networks context.

\section{Acknowledgements}

This work has been developed within the research project called "Exploring latent information of key performance indicators by identifying their cause-effects relationships at the inter-enterprise context (1992)" funded by the Universitat Politècnica de València.

\section{References}

1. Matopoulos, A., Vlachopoulou, M., Manthou, V., Manos, B.: A conceptual framework for supply chain collaboration: empirical evidence from the agri-food industry. Supply Chain Management: An International Journal, 12, 3, 177-186 (2007)

2. Barrat, M.: Understanding the meaning of collaboration in the supply chain. Supply Chain Management: An International Journal, 9 (1), 30-42 (2004)

3 Alfaro, JJ., Rodriguez-Rodriguez, R., Verdecho, MJ., and Ortiz, A.: Business process interoperability and collaborative performance measurement. International Journal of Computer Integrated Manufacturing, Vol. 22, No. 9, 877-889 (2009)

4 Franco, R.D. and Ortiz, A.: Soporte a la ejecución de procesos de negocio extendidos para la planificación de la producció. Proceedings of the X Congreso de Ingeniería de Organización, Valencia, I, 255-262 (2006)

5 Ghalayini, A., Noble, J.S. and Crowe, T.J.: An integrated dynamic performance measurement system for improving manufacturing competitiveness. International Journal of Production Economics, 48, pp. 207-225 (1997)

6 Waggoner, D., Neely, A.D. and Kennerley, M.P.: The forces that shape organisational performance measurement systems: An interdisciplinary review. International Journal of Production Economics, 60 (61), pp. 53-60 (1999)

7 Bititci, U.S., Turner, T. and Begemann, C.: Dynamics of performance measurement systems. International Journal of Operations and Production management, 20 (6), pp. 692-704 (2000)

8 Kennerley, M. and Neely, A.: Measuring performance in a changing business environment. International Journal of Operations \& Production Management, 23 (2), pp. 213-229. (2003)

9 Kennerley, M., Neely, A. and Adams, C.: Survival of the fittest: measuring performance in a changing business environment. Measuring Business Excellence, 7 (4), pp. 37-43. (2003)

10 Najmi, M., Fan, I. and Rigas, J.: A framework to review performance measurement systems. Business Process Management Journal, 11 (2), p.109-122. (2005)

11 Salloum, M.: Towards dynamic performance measurement system: a framework for manufacturing organizations. Thesis, Mälardalen University, Västeras, Sweden (2011) 